\title{
In situ measurement of internal stresses and strain rates by high energy $X$-Ray diffraction during high temperature mechanical testing
}

\author{
A Jacques ${ }^{1}$, L Dirand ${ }^{1}$, J P Chateau', T Schenk ${ }^{1}$, O Ferry ${ }^{1}$ \\ and $\mathrm{P}$ Bastie $^{2}$ \\ ${ }^{1}$ Institut Jean Lamour (SI2M) (UMR CNRS-Nancy Université N ${ }^{\circ} 7198$ ) Ecole des \\ Mines, parc de Saurupt 54042 Nancy cedex, France. \\ ${ }^{2}$ Laboratoire de Spectrométrie Physique, (UMR CNRS-Université Joseph-Fourier \\ $N^{\circ}$ 5588), BP 87, 38402 Saint-Martin-d'Hères cedex, France \\ alain.jacques@mines.inpl-nancy.fr, laura.dirand@mines.inpl-nancy.fr, jean- \\ philippe.chateau@mines.inpl-nancy.fr, thomas.shenk@mines.inpl-nancy.fr, \\ olivier.ferry@mines.inpl-nancy.fr, bastie@ill.fr
}

Key words : Nickel-based superalloys, X-ray diffraction, Three Crystal Diffractometry (TCD), mechanical properties, in situ experiments

\begin{abstract}
The combination of high temperature $\left(1050^{\circ} \mathrm{C}-1150^{\circ} \mathrm{C}\right)$ testing and in situ high energy $\mathrm{X}$-Ray diffraction measurements using synchrotron Three Crystal Diffractometry may give various insights into the mechanical behaviour of superalloys: measurement of the lattice mismatch, order within the $\gamma^{\prime}$ phase, elastic constants, and dynamic response to changes in the experimental conditions. Several examples are given on the rafted AM1 superalloy, resulting from experiments at the ID15A (ESRF) and BW5 (DESY) high energy beamlines.
\end{abstract}

\section{Introduction}

During the second stage of their high temperature tensile creep curve ([001] tensile axis), rafted single crystalline nickel-based superalloys may be seen as very simple two-phased materials: they are formed of alternate, semi coherent, layers perpendicular to the tensile axis of a $\mathrm{L}_{2}\left(\gamma^{\prime}\right)$ phase (so called rafts) and of a disordered fcc $\gamma$ phase ( $\gamma$ corridors). This simplicity might make superalloys obvious candidates for physically-based modelling of their plastic behaviour, taking into account dislocation velocities, densities..., and internal stresses. However, in spite of very sophisticated attempts [1,2], this has not yet been done, as experimental data are lacking. A main parameter [3] is the lattice mismatch $\delta$ :

$$
\delta=2\left(a_{\gamma^{\prime}}-a_{\gamma}\right) /\left(a_{\gamma^{\prime}}+a_{\gamma}\right) .
$$

where $a_{\gamma^{\prime}}$ and $a_{\gamma}$ are the lattice parameter of the $\gamma^{\prime}$ and $\gamma$ phase respectively. The value of $\delta$ may reach a few $10^{-3}$, and depends on the exact chemical composition of the phases and on temperature. Its sign and value have a major impact on the transition, during stage I of creep, from the initial microstructure with cuboids to the rafted one. Experimental data on the elastic constants (Young modulus $\mathrm{E}_{\gamma}$ and $\mathrm{E}_{\gamma^{\prime}}$ ) are also scarce, as their measurement with classical methods at a given temperature would require growth of single phase, single crystalline specimens with the exact equilibrium composition of the $\gamma$ and $\gamma^{\prime}$ phases at that temperature.

While these parameters remain constant during a test, we may however expect dislocation densities, the level of internal stress, and even the fraction $f$ of $\gamma^{\prime}$ phase to vary on timescales of one hour or less. If the lattice mismatch is partly relaxed by the dislocation array left at the $\gamma / \gamma^{\prime}$ interfaces by dislocation which moved within the $\gamma$ corridors and were not transmitted into the rafts, we have no reason to suppose they are cancelled at equilibrium, or that they remain constant while the corridor width increases with time, or when the external stress is changed. 
TEM observations made post mortem on specimens cooled down quickly, in order to freeze the microstructure, will allow measuring dislocation densities, [4] or internal stresses [5] but at one point only. In situ TEM experiments [6] may give valuable insights on the dislocation mechanisms, but may require a careful extrapolation from a thin foil to bulk material.

An experimental method which would allow stress and strain measurements on bulk materials during a realistic test, with a time resolution of a few hundred seconds or less might thus give invaluable input data for modelling of the plastic behaviour of superalloys. The aim of the present paper is to show that in situ experiments using Three Crystal Diffractometry (TCD) with hard Synchrotron X-Rays [7] might give some of the required data. After a brief description of the method, of the resulting data and their processing, a few examples of its use will be given on the AM1 superalloy.

\section{Three Crystal Diffractometry.}

Figure 1 shows a sketch of the diffraction setup. An incident white X-Rays beam is first diffracted in transmission geometry by a silicon monochromator. The monochromatized beam is then diffracted by different sets of lattice planes of the specimen into beams having slightly different orientations. A second silicon crystal, termed analyzer, selects one of these beams, and sends it to an energy selective Ge detector.

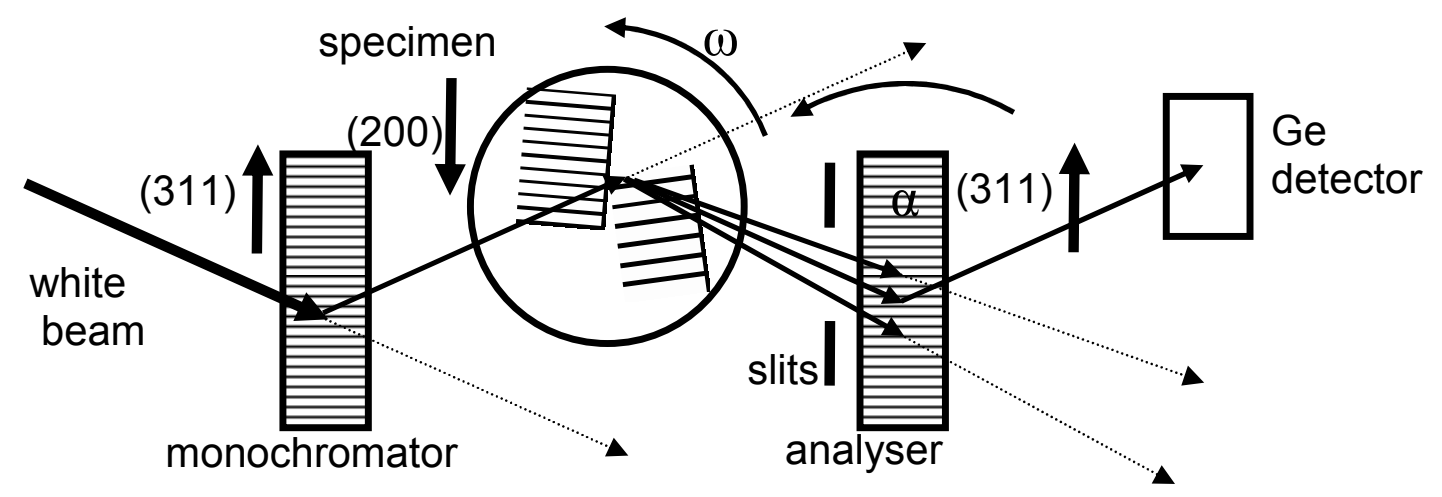

Fig.1 Setup of the Three Crystal transmission Diffractometer with two (311) Si single crystals as monochromator and analyzer.

A rotation of the specimen angle $\omega$ only scans through the orientation of the reflecting plane. (As single crystalline superalloys are not perfect, these orientations may span a fraction of a degree.) A rotation of the analyzer ( $\alpha$ scan) will select beams diffracted by sets of specimen lattice planes having slightly different orientations and interplanar distance (range: a few $10^{-3}$ ). A $\theta-2 \theta$ scan will rotate both angles, with $\mathrm{d} \alpha=2 \mathrm{~d} \omega$. The beams transmitted to the detector will have been diffracted by lattice planes of the specimen having all the same normal and different spacings. Their intensity will be proportional to the density of the diffracting lattice planes.

The choice of a high energy monochromatic beam $(120 \mathrm{keV}$ to $150 \mathrm{keV}$ range, i.e. $\lambda=0.01 \mathrm{~nm}$ to $0.008 \mathrm{~nm}$ ) will allow to operate in transmission geometry through a specimen with a $3 \mathrm{~mm}$ diameter, and to scan lattice parameters within the bulk of the specimens. The choice of (311) reflections (interplanar distance: $0.1637 \mathrm{~nm}$ ) for the monochromator and analyzer and a (200) reflection $(\mathrm{d} \approx 0.178 \mathrm{~nm})$ for the specimen and a $(+,-,+)$ geometry results in a very low contribution $(<0.6$ second of arc) of the setup to the width of the peaks. The high beam intensity provided by synchrotron sources such as ID15 (ESRF) and BW5 (DESY) and the quality of the diffractometers allows the recording of a 200 points scan in $\approx 300$ s, with a good statistics even on the peak tails.

Fig. $2 \mathrm{a}$ shows the results of an $\omega$ scan, and Fig. $2 \mathrm{~b}$ the result of a $\theta-2 \theta$ scan recorded at $120 \mathrm{keV}$, at room temperature, on a plastically strained single crystalline Ni3Al specimen. The three peak distribution of the $\omega$ scan results from subgrain boundaries within the gauge length. The $\theta-2 \theta$ scan mainly consists of a Gaussian peak (std. deviation: 8.4 second of arc) with a small Lorenzian tail, and an asymmetric background (10 cts on the right, and $90 \mathrm{cts}$ on the left). The higher background 
on the low $2 \theta$ side is thought to result from a low energy tail in the photon energy distribution, perhaps due to double Compton scattering within the specimen. (Only 38\% of the initial beam intensity was transmitted through this specimen.) The beam intensity is cut on both sides by the 100 $\mu \mathrm{m}$ slits which define the beam. Figs. $2 \mathrm{c}$ and $2 \mathrm{~d}$ show an $\omega$ scan, and the two-peaked distribution measured during $\theta-2 \theta$ scans on rafted specimens, with a (200) diffraction vector (perpendicular to the tensile axis). The surface of the $\gamma^{\prime}$ peak is $65 \%$ of the whole area. The distance between both peaks is 33.3 seconds of arc, corresponding to a -0.0033 value of $\delta \perp$, the lattice mismatch measured in the [100] direction. The peak width is slightly lower than that of the single crystal. An important feature is the linear slope (observed on all superalloys specimens) of both peaks in logarithmic scale with slopes $1 / \mathrm{w}$ and $1 / \mathrm{w}^{\prime}$ respectively. The asymmetric background is lower, but still present.

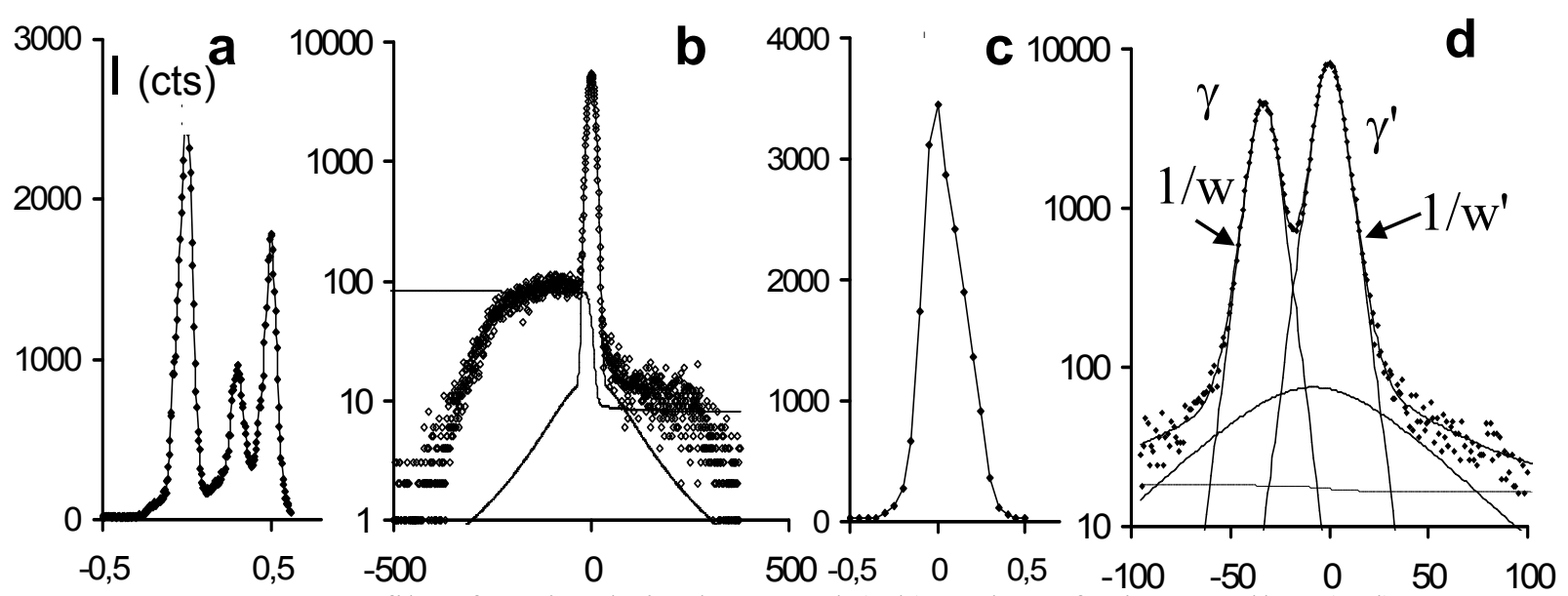

Fig.2 Diftraction profile of a Ni3Al single crystal (a,b) and a rafted superalloy (c,d). a,c: $\omega$ scan (units: degrees). b,d: $\theta-2 \theta$ scan (seconds of arc, and logarithmic scale for I ).

\section{Temperature effect on the lattice mismatch and order of the $\gamma^{\prime}$ phase.}

Fig. 3 shows the variations of the lattice mismatch recorded along [100] $\left(\delta_{\perp}\right)$ and [001] $\left(\delta_{/ /}\right)$for two specimens with a rafted microstructure. As long as the dislocation array at the $\gamma / \gamma^{\prime}$ interfaces remains unchanged, $\delta_{\perp}$ is nearly constant and $\delta_{/ /}$follows the changes in $\delta$. Assuming that the ratio between the Young modulus of both phases is also constant, $\delta_{/ /}$does not depend on the volume fraction $\mathrm{f}$ of the $\gamma^{\prime}$ phase and can be written as [8]:

$$
\delta=\frac{1-v}{1+v} \delta_{/ /}+\frac{2 v}{1+v} \delta_{\perp} .
$$

where $v=0.43$ is the Poisson coefficient.
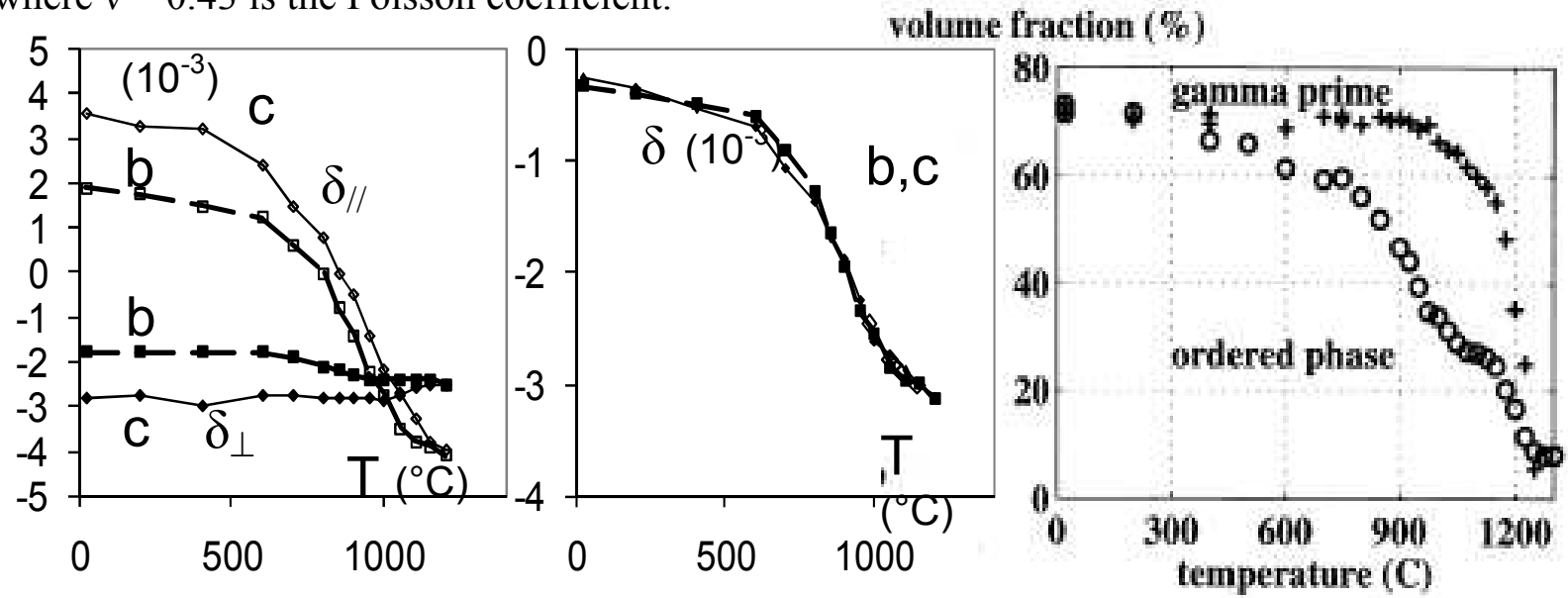

Fig.3 a; Lattice mismatch of two rafted AM1 specimens measured along $\left(\delta_{/ /}\right)$and perpendicular $\left(\delta_{\perp}\right)$ to the tensile axis [9]. b; "natural" mismatch deduced from these measurements. c; Evolution of the area of the $\gamma^{\prime}$ peak and the superstructure (100) peak. 
As the relative area of each peak is related to the volume of each phase, the evolution of the volume fraction $\mathrm{f}$ of the $\gamma^{\prime}$ phase with temperature can be determined [10]. Furthermore superstructure reflections are observed in superalloys. They are related to ordering inside the fcc structure. As the $\gamma^{\prime}$ phase is the only part of the material which possesses an order, the intensity of the superstructure reflections, like (100) reflection, is related both to the order and to the volume fraction of the $\gamma$ ' phase. It is observed on Fig. 3c that the two quantities do not evolve in the same way with temperature. Above $500-600^{\circ} \mathrm{C}$ the $\gamma^{\prime}$ phase is not fully ordered. As the $\mathrm{L}_{2}$ order explains some of its properties (pairing of dislocations, AntiPhase Boundaries), this should influence the mechanical behavior of the rafts.

\section{Elastic constants.}

The peak positions measured along [100] shift to larger angles when the tensile load $\sigma$ is increased. When the density of dislocations at the $\gamma / \gamma^{\prime}$ interfaces remains constant, this shift (i.e. the variations of $\varepsilon_{\mathrm{er}}$ and $\varepsilon_{\mathrm{e} \gamma}$ ) must be the same for both phases. When new dislocations come at the interface or move from the interface to the $\gamma^{\prime}$ rafts, $\delta_{\perp}$ changes, and the additional elastic strain is shared unevenly between both phases, depending on the $\gamma^{\prime}$ volume fraction $f$ and Young modulus $E$ and $E^{\prime}$. This may be summarized as [11]:

$$
\begin{aligned}
& \Delta \varepsilon_{e \gamma}=-\chi \cdot \Delta \delta_{\perp}-v\left(\chi / E^{\prime}+(1-\chi) / E\right) \sigma . \\
& \Delta \varepsilon_{e \gamma^{\prime}}^{\prime}=(1-\chi) \cdot \Delta \delta_{\perp}-v\left(\chi / E^{\prime}+(1-\chi) / E\right) \sigma . \\
& \text { Where: } \left.\chi=f E^{\prime}\left[f E^{\prime}+(1-f)\right) E\right]^{-1} .
\end{aligned}
$$

A fit of these variations is given in Fig. 4, for an AM1 specimen strained at $1090^{\circ} \mathrm{C}$. Taking $\mathrm{f}=$ 0.627 from the relative area of the peaks and $v=0.43$, the best fit gives $\mathrm{E}=48 \mathrm{GPa}$ and $\mathrm{E}^{\prime}=75 \mathrm{GPa}$ at $1090^{\circ} \mathrm{C}$. A slight discrepancy $\left(\approx 5.10^{-5}\right)$ remains at low loads, which requires further investigations.

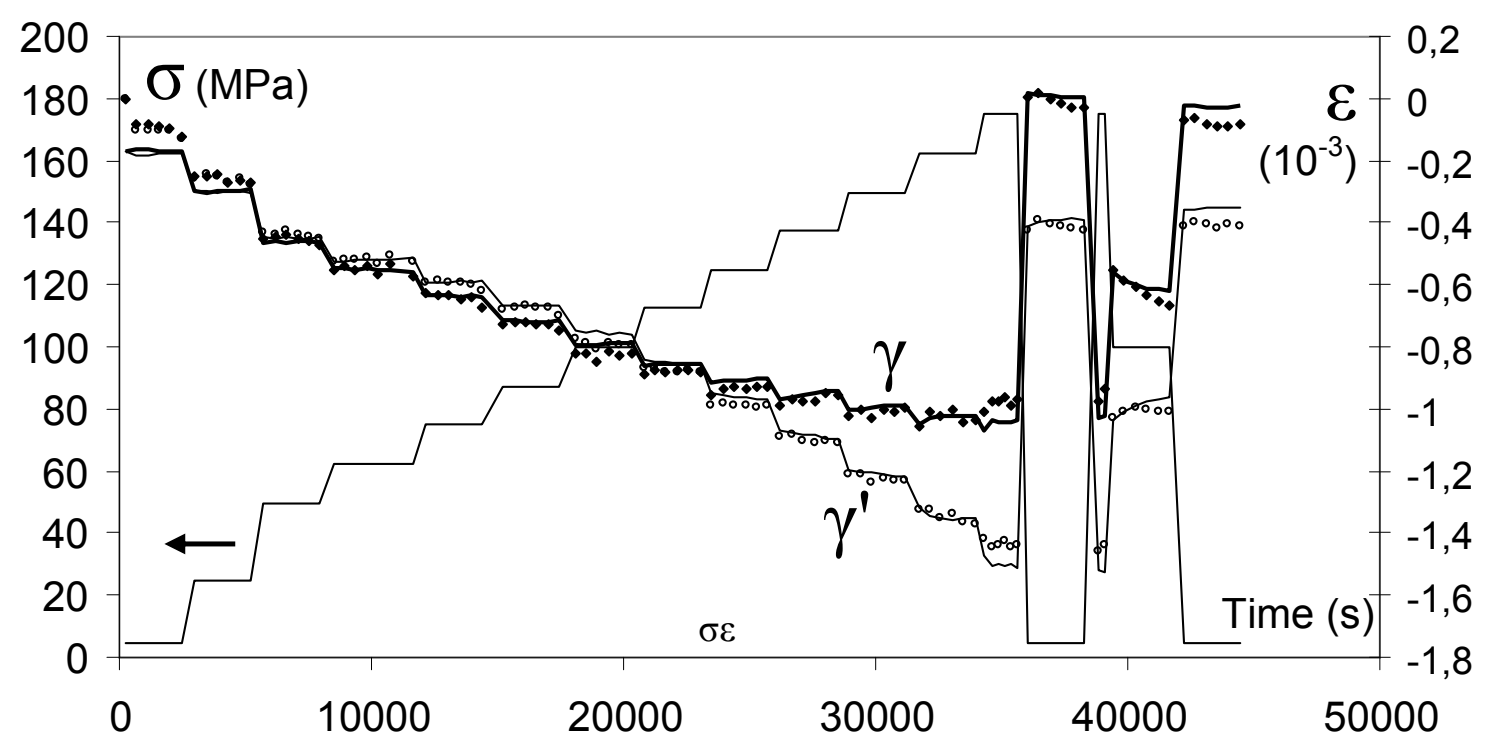

Fig.4 Fit of the relative variation of the lattice parameters (i.e. elastic strains, right angle scale) perpendicular to the tensile axis during a loading/unloading sequence. $\left(1090^{\circ} \mathrm{C}\right)$ 


\section{Dynamical behaviour.}

After loading steps at high loads $(\sigma>120 \mathrm{MPa}$, see Fig.5), new dislocations of the slip systems having a non-zero Schmid factor can move into the $\gamma$ corridors. These dislocations leave dislocation segments trapped along the intersection between their slip plane and the $\gamma / \gamma$ ' interfaces. The Burgers vector of all these segments have a component in the (001) plane, which will tend to decrease the algebraic value of $\delta_{\perp}$. As a result, the effective stress within the corridors (applied stress + internal stress) on dislocations of the same slip system also decreases, while it increases within the rafts. This dislocation movement stops when the effective stress has reached the threshold value for entering the corridors (Orowan stress), and $\delta_{\perp}$ has found a new equilibrium value. During constant successive loading steps of the specimens, the $\gamma$ corridors are thus submitted to successive loading and relaxation cycles, i.e. to successive relaxation tests, with a constant plastic stain increment. As long as the plastic strain rate of the rafts remains low and can be neglected, the variation with time of $\delta_{\perp}$, or the distance $\theta-\theta^{\prime}$ between peaks, can be used to investigate the relaxation kinetics. The fits of the variation of $\theta-\theta^{\prime}$ with time in Fig. 5a show that the relaxation is exponential, and that the relaxation time $\tau$ decreases with the step number. The only variable parameter of the microstructure is the density of mobile dislocations $\rho_{\mathrm{m}}$, which must increase. The plot in Fig. $5 \mathrm{~b}$ indeed shows that $1 / \tau$ (proportional to $\rho_{\mathrm{m}}$ ) seems to be proportional to the step number, i.e. to the cumulated strain of the rafts.
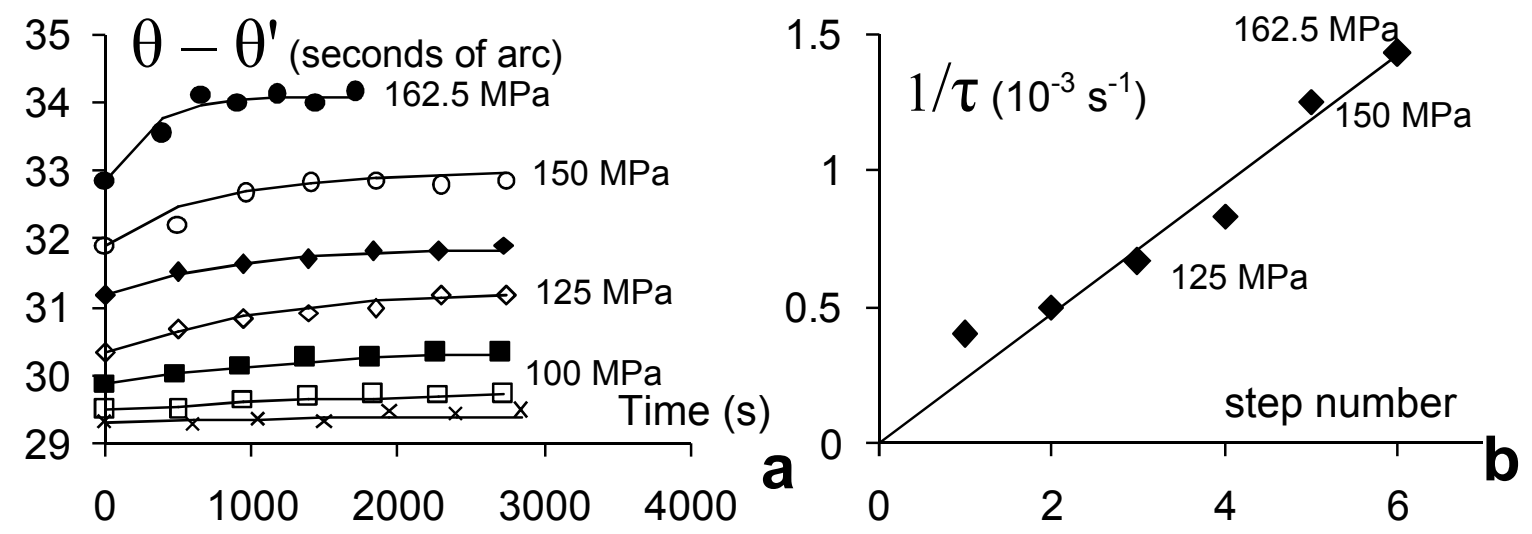

Fig.5 a; Fit of variation of the peak distance with time for successive $12.5 \mathrm{MPa}$ steps. b; Variation of $1 / \tau$ with the step number. $\left(\mathrm{AM} 1,1090^{\circ} \mathrm{C}\right)$

\section{Conclusion}

As shown in these few examples, the combination of precise measurements of the shape of diffraction peaks with in situ high temperature testing can be a very powerful technique to measure "static" data such the elastic constants or the lattice mismatch. More, it is possible to follow the dynamics of plasticity on time scales of a few hundred seconds, on a representative volume $(\approx 0.3$ $\mathrm{mm}^{3}$ ) of bulk specimens. Such features are complementary of those of Transmission Electron Microscopy, for observations done either post mortem or in situ. The timescale could be shortened with current progress both in the power of new synchrotron sources and detectors, and could make it suitable to study the transient behavior of superalloys under extreme conditions, which is critical for aircraft under heavy use conditions.

\section{Acknowledgements}

The authors would like to thank the DESY (BW5 beamline) and ESRF (ID15 beamline) staff for their help in the experiments, and P. Caron (ONERA) and Y. Guédou (SNECMA) who provided the specimens. Funding from the European Community's Seventh Framework Programme (FP7/20072013) under grant agreement $n^{\circ} 226716$ is acknowledged. 


\section{References}

[1] B. Fedelich: Int. Jour of Plasticity Vol. 18 (2002), p.1

[2] I. N. Vladimirov: Int. Jour. of Mech. Sciences Vol 51 (2009), p. 305

[3] H. Biermann, M. Strehler, and H. Mughrabi: Met. Mater. Tr. A Vol. 27 (1996), p. 1003.

[4] J.X. Zhang,T. Murakumo, H. Harada,Y. Koizumi Scripta Mater. Vol. 48 (2003), p. 287

[5] Z. Lu, F. Pyczak, H. Biermann, and H. Mughrabi: Phil. Mag. A, Vol. 82, (2002), p.1219

[6] M. Benyoussef, N. Clément, and A. Coujou: Phil. Mag. A, Vol. 72 (1995), p. 1043.

[7] A. Royer, P. Bastie: Scripta. Mater. Vol. 36 (1997), p. 1151

[8] A. Jacques and P. Bastie: Phil. Mag. A Vol. 83 (2003), p. 3005

[9] A. Royer, P. Bastie, M. Veron: Acta Mater. Vol 46 (1998), p. 5357

[10] A. Royer, P. Bastie, and M.Véron: Scripta Mater. Vol. 40 (1999), p.955

[11] A. Jacques, F. Diologent, P. Caron, P. Bastie: Mat. Sci. Eng. A Vol. 483 (2008), p.568 